\title{
Measuring Drivers' Physiological Response to Different Vehicle Controllers in Highly Automated Driving (HAD): Opportunities for Establishing Real-Time Values of Driver Discomfort
}

\author{
Vishnu Radhakrishnan ${ }^{1}{ }^{*} \mathbb{1}$, Natasha Merat ${ }^{1}$, Tyron Louw ${ }^{1}$, Michael G. Lenné ${ }^{2}$, \\ Richard Romano ${ }^{1}$, Evangelos Paschalidis ${ }^{1}$, Foroogh Hajiseyedjavadi ${ }^{1}$, Chongfeng Wei ${ }^{1} \mathbb{C}$ \\ and Erwin R. Boer ${ }^{3}$ \\ 1 Institute of Transport Studies, University of Leeds, Leeds LS29JT, UK; N.Merat@its.leeds.ac.uk (N.M.); \\ T.L.Louw@leeds.ac.uk (T.L.); R.Romano@leeds.ac.uk (R.R.); E.Paschalidis@leeds.ac.uk (E.P.); \\ F.Hajiseyedjavadi@leeds.ac.uk (F.H.); chongfeng.wei@northumbria.ac.uk (C.W.); \\ 2 Seeing Machines Ltd., Canberra 2609, Australia; mike.lenne@seeingmachines.com \\ 3 Entropy Control, Inc., San Francisco, CA 94107, USA; erwinboer@entropycontrol.com \\ * Correspondence: mn16vr@leeds.ac.uk; Tel.: +44-74-69318182
}

Received: 2 July 2020; Accepted: 5 August 2020; Published: 8 August 2020

\begin{abstract}
This study investigated how driver discomfort was influenced by different types of automated vehicle (AV) controllers, compared to manual driving, and whether this response changed in different road environments, using heart-rate variability (HRV) and electrodermal activity (EDA). A total of 24 drivers were subjected to manual driving and four AV controllers: two modelled to depict "human-like" driving behaviour, one conventional lane-keeping assist controller, and a replay of their own manual drive. Each drive lasted for $~ 15$ min and consisted of rural and urban environments, which differed in terms of average speed, road geometry and road-based furniture. Drivers showed higher skin conductance response (SCR) and lower HRV during manual driving, compared to the automated drives. There were no significant differences in discomfort between the AV controllers. SCRs and subjective discomfort ratings showed significantly higher discomfort in the faster rural environments, when compared to the urban environments. Our results suggest that SCR values are more sensitive than HRV-based measures to continuously evolving situations that induce discomfort. Further research may be warranted in investigating the value of this metric in assessing real-time driver discomfort levels, which may help improve acceptance of AV controllers.
\end{abstract}

Keywords: driver state; discomfort; psychophysiology; heart-rate variability (HRV); skin conductance response (SCR); highly automated driving (HAD)

\section{Introduction}

In the recent past, there has been an increasing interest in implementing vehicles with a range of advanced driver assistant systems (ADAS), fuelled by manufacturers' desire to introduce higher levels of vehicle automation capability [1]. The primary motivation for these implementations is their hypothesised provision of increased road safety, and enhanced mobility, accessibility, efficiency and comfort [2]. According to Carsten and Mertens [3], manufacturers have been using comfort as one of the main selling points for ADAS. Additionally, the comfort of the driver is considered to be a determining factor for the broader acceptance of the automated system [4]. Therefore, it can be argued that, if an automated system can measure driver comfort in real-time, it can adapt its driving style/behaviour to match the drivers' expectations accordingly, and thereby potentially increase 
acceptance. This could have the additional benefit of reducing unnecessary driver initiated takeovers, which can otherwise jeopardise the safety of the vehicle and its occupants [5]. This study, conducted as part of the HumanDrive project, considered the effect of a number of road and vehicle-based factors on driver comfort, investigating whether physiological metrics can be used to provide an objective measure of comfort, to help inform the design process when investigating the acceptance of future automated vehicles.

Currently, there is no unanimously agreed on definition of comfort. In a general context, Slater [6] (p. 158) described comfort as "a pleasant state of physiological, psychological and physical harmony between human being and the environment". In the context of driving, and especially highly automated driving (HAD), Beggiato et al. [7] (p. 446), defined comfort as "a subjective, pleasant state of relaxation resulting from confidence in safe vehicle operation which is achieved by the absence of uneasiness and distress". Beggiato et al. [7] further suggested this is still a rather broad definition of comfort, and is associated with other concepts, such as stress, mental workload, fear, motion sickness or anger, with stress and mental workload having the closest link to discomfort (i.e., lack of comfort). Siebert et al. [4] argued that it is easier to measure discomfort rather than comfort, since signs of discomfort tend to be more well-defined and pronounced, compared to the un-aroused relaxed state of comfort. Summala [8] proposed four factors that need to be maintained above a certain threshold to keep drivers within their "comfort zone" during manual driving. These are safety margins (to road edges, obstacles or other vehicles), vehicle-road system (accelerations, road geometry), rule-following (obeying traffic laws, maintaining speed limits) and good progress of the trip (meeting one's expectations for the pace or progress of the travel). However, assuming 100\% performance of the automated system, Siebert et al. [4] noted that the rule-following factor for comfort is redundant in HAD, as the automated vehicle (AV) will almost certainly follow the rules, and that good progress of the trip is dependent on traffic conditions, rather than automation state in itself, assuming the route selected by the AV is similar to that in manual driving, where the navigation system decides/recommends the optimal route to be followed. Therefore, in this paper, we focus specifically on how factors that affect the safety margins, and vehicle-road system, affect driver discomfort, for manual and automated driving.

Summala [8], suggested that sufficient safety margins from potential hazards are required for a driver to feel safe and comfortable. Factors influencing these safety margins, and likely to increase driver discomfort, include situations which increase drivers' stress levels, such as navigating in crowded cities, interactions with other road users, or when passing another car/obstacle [9,10].

Comfort is affected by jerk and acceleration forces of the vehicle, with higher accelerations and jerks (in terms of both magnitude and frequency) associated with an increase in discomfort [11-13], and an increase in motion sickness [14]. Drivers tend to keep their lateral and longitudinal acceleration under $2 \mathrm{~m} / \mathrm{s}^{2}$ for a comfortable driving experience [15-17]. However, it should be noted that drivers' comfort threshold for lateral acceleration varies with respect to their velocity, with an increase in velocity resulting in lower threshold values for lateral acceleration $[17,18]$. Within the public transport domain, especially in railway systems, standard acceleration values are limited to under $1.47 \mathrm{~m} / \mathrm{s}^{2}$, and jerk values are kept under $0.6 \mathrm{~m} / \mathrm{s}^{3}$, to ensure passenger comfort $[13,16,19]$. However, the acceleration and jerk thresholds used in public transport systems consider both seated and standing passengers. Therefore, it may be permissible to have slightly higher thresholds in HAD, where passengers are typically seated. For instance, Eriksson and Svensson [20] suggested an acceleration and jerk threshold of under $2 \mathrm{~m} / \mathrm{s}^{2}$ and $0.9 \mathrm{~m} / \mathrm{s}^{3}$ respectively, to ensure a comfortable ride in HAD.

Because AVs are still in the prototype and testing phase, most individuals have not had a real-world experience of HAD. Therefore, our expectations of what constitutes a 'comfortable' experience during HAD can only be based on our current understanding of users' comfort in either manual driving, or in other surface transport modes. However, there are considerable differences between these modes, in terms of Summala's [8] proposed four factors, described above, making them difficult to compare to HAD. Thus, to assist with the development of more acceptable AVs, and to ensure user uptake of these systems in the future, it is of value to understand what particular features of an AV's manoeuvres 
are likely to enhance or diminish user discomfort. For example, humans try to minimise the jerk during manual driving, whereas most current ADAS features tend to have a relatively higher jerk, due to their preference to stay closer to the lane centre and unwillingness to cut corners, unlike human drivers. Thus, it is important to know if users would prefer, and feel more comfortable with, a more "human-like" AV controller, which favours manoeuvres that result in lower acceleration and jerk, over a more conventional AV controller, with very strict margins for optimal and accurate lane-keeping and vehicle velocities.

Studies on comfort in manual driving have used subjective measures, such as comfort questionnaires [21] and comfort scales [22]. Since comfort is highly subjective, it can be challenging to measure it accurately and reliably on a moment-to-moment basis. In a real-world HAD scenario, the driver may become annoyed if they are asked to rate their comfort levels time and again during the drive, especially when they have the option to engage in more appealing non-driving related activities. Thus, in HAD, there is a need for a non-intrusive, objective, discomfort detection system, which can ultimately be used to adapt the automated system's driving style, to ensure the driver is relaxed and at ease [7]. Physiological techniques are one example of such objective methods, which have been used in the past to assess driver state both in HAD [7] and manual driving [23,24]. Recent technological advancements have led to the development of non-intrusive physiological devices that measure heart rate variability (HRV) and electrodermal activity (EDA), such as wearable smart-band sensors like Empatica E4 [25] or Microsoft band 2 [7], and non-contact methods, such as those listed in [26]. Previously, studies have shown strong correlations between stress and workload, and users' HRV, and EDA. A general finding is that heart rate (HR) increases, and HRV (including the time-domain based metric of root mean square of successive differences in R-R intervals (RMSSD)) decreases, during periods of high stress or workload [10,27-29].

An EDA signal consists of the slow-changing tonic component called skin conductance level (SCL) and the rapidly changing phasic component, known as skin conductance response (SCR) [30]. SCRs are generally used to understand short-term fluctuations in the EDA signal, due to a short-term stimulus (for example, being startled or passing an obstacle), whereas SCL is used to understand the overall change in a person's skin conductance when the stimulus is spread over a longer period (for example, fatigue induced by driving for a long time). SCRs have a much shorter decay time than SCLs, and, hence, can more accurately capture differences in manipulations, without the need for recovery/resting periods in between [30,31]. In the context of driving, both SCL and SCRs have been shown to increase with an increase in stress and workload for a driver [10,23,32], and, thus, are associated with increases in discomfort [7]. Based on these findings, we analysed RMSSD, HR and SCR responses per minute $(\mathrm{nSCR} / \mathrm{min})$ in this study, as the objective physiological metrics of drivers' comfort.

\section{Current Study}

This study was undertaken as part of a 10-member consortium of the HumanDrive project, part-funded by the UK's Centre for Connected and Autonomous Vehicles (CCAV), via Innovate UK. The main aim of the project was to develop an advanced vehicle controller, which allowed the vehicle to perform a 'natural', human-like, driving style, using artificial intelligence (AI), and deep learning techniques. As outlined above, developing a human-like controller could potentially help with the broader acceptance of AVs, driven by a more natural driving style, which is familiar to the driver. Using manual driving data collected from 44 drivers in an earlier HumanDrive study, an aggregated model for human-like controllers, focusing on both vehicle safety and comfort, was developed for the present study (see also [33], for more details of the controllers). An environment-specific risk model was developed to guide the design of the experiments. The simulated drives were constructed to include risk elements present in the drive, based on road width and curvature, as well as on the presence of road-based furniture and obstacles, such as hedges of different heights, grass/asphalt verges, pedestrian refuges and parked-cars or roadworks (see [34] for more details). The development 
of this risk model was based on satisficing risk corridors, proposed by Boer [35], where a set of vehicle states are within acceptable bounds. The vehicle state includes velocity and lateral offset. The trajectory of the vehicle is always within this risk corridor and adopts a comfortable smoothness for the ride. The model holds that drivers' perceived risk level is based on minimum time to lane crossing, wherein the lateral position for the vehicle stays within the road boundaries [35]. Based on this model, two human-like AV controllers (SLOW and FAST, with the FAST controller having higher velocities than the SLOW controller) were developed, and compared to a conventional controller (LKAS), and drivers' replay of their own drive (see Section 2.3, for more details). To understand how the different physical characteristics of a drive can affect drivers' discomfort, our study exposed participants to a range of accelerations, induced by the four different AV controllers and manual driving. Participants experienced these controllers in two different road environments (rural and urban), which included a variety of road geometries, such as roads of different curvatures/width/speed limit, containing a range of road furniture/obstacles (parked cars, roadworks and pedestrian refuges). Previous studies on driver discomfort during HAD, such as Beggiato et al. [7], have focused on discrete situations causing discomfort, such as negotiating an intersection, exit ramp or an obstacle. In our study, we considered the effects of longer, repeated exposure to different road environment, human-like $\mathrm{AV}$ controllers and interactions with road furniture and obstacles, on drivers' discomfort. Drivers' HR and EDA data were compared to drivers' self-reported level of perceived discomfort for each road environment, which was measured in real-time, using a button pressing technique (see Section 3.2 for more details). We addressed the following research questions:

i. How is driver discomfort, as measured by changes in physiological state (i.e., HRV and EDA), affected by the various controllers, and manual driving?

ii. Do drivers' discomfort levels change, based on the behaviour of the different controllers, in the different road environments (rural and urban)?

iii. Does the change in drivers' physiological state reflect their self-reported level of perceived discomfort during HAD?

\section{Materials and Methods}

\subsection{Participants}

In total, 24 participants (10 Female), each with a valid UK driving licence, took part in this driving simulator-based study. Their mean age was $43 \pm 17$ years, with a mean driving experience of $23 \pm 18$ years. All participants gave consent to take part in the study, in accordance with the rules and regulations of the University of Leeds ethics committee (LTTRAN-086) and were compensated with $£ 50$ for taking part in the study. Participants were pre-screened for physiological data collection and those with pre-existing heart conditions were not included in the study (as per [30,36]). In addition, participants were requested to avoid consuming food and beverages that had cardiac stimulants such as caffeine or alcohol for $24 \mathrm{~h}$ before they took part in the study.

\subsection{Aparatus}

The experiment was conducted in the full motion-based University of Leeds Driving Simulator (UoLDS), which consists of a Jaguar S-type cab housed in a $4 \mathrm{~m}$ diameter spherical projection dome with a 300-degree field-of-view projection system. The simulator also incorporates an 8 degree-of-freedom electrical motion system. This consists of a $500 \mathrm{~mm}$ stroke-length hexapod motion platform, carrying the $2.5 \mathrm{~T}$ payload of the dome and vehicle cab combination, and allowing movement in all six orthogonal degrees-of-freedom of the Cartesian inertial frame. Additionally, the platform is mounted on a railed gantry that allows a further $5 \mathrm{~m}$ of effective travel in surge and sway. Drivers' physiological data were collected using a Biopac MP35 data acquisition system at $1000 \mathrm{~Hz}$, which consisted of ECG electrodes and an EDA sensor. 


\subsection{Study Design}

The study used a within-participant design and included a short familiarisation drive for $\sim 10 \mathrm{~min}$. Each participant experienced five drives: a MANUAL drive, two with human-like AV controllers (SLOW and FAST), a replay of their manual drive (REPLAY) and one conventional lane-keeping assist-based AV controller (LKAS) which did not adapt its behaviour to road furniture, such as kerbs or hedges. Each drive consisted of two different road environments (rural and urban). The design of the drives and the road environments are discussed below.

\subsubsection{Road Design}

Each drive was $15.8 \mathrm{~km}$ long, and incorporated several situations that demanded greater attention and a shift in lateral position and speed, which could be deemed uncomfortable by the driver based on how it was negotiated, presented across two different road environments (rural and urban, see Figure 1). The speed limits, geometries, and obstacle locations, for each road are listed in Table 1 and Figure 2. The road design was similar across all drives except for LKAS, which did not include any obstacles, which were partly within the lane, such as roadworks or parked cars.

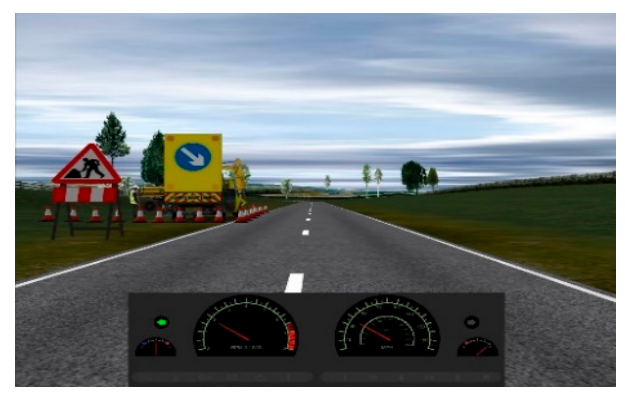

(a)

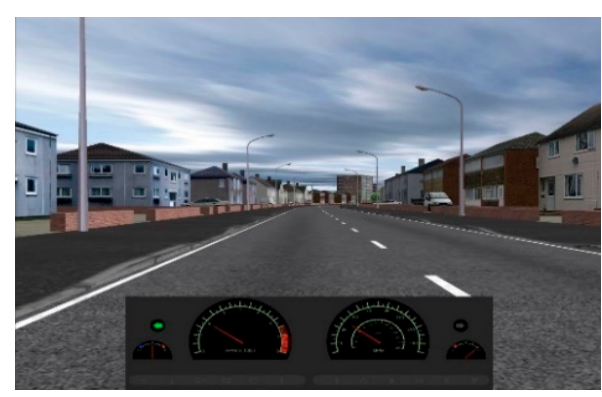

(b)

Figure 1. (a) Rural environment with roadworks; (b) urban environment.

Table 1. Road geometry and furniture across different segments (in the order they were experienced).

\begin{tabular}{|c|c|c|c|c|c|c|c|c|}
\hline \multirow{2}{*}{$\begin{array}{l}\text { Segment } \\
\text { Segment } 1\end{array}$} & \multirow{2}{*}{$\begin{array}{c}\text { Obstacles } \\
-\end{array}$} & \multirow{2}{*}{$\begin{array}{c}\text { Environment } \\
\text { rural }\end{array}$} & \multirow{2}{*}{$\begin{array}{c}\begin{array}{c}\text { Speed Limit } \\
\text { (mph) }\end{array} \\
60\end{array}$} & \multirow{2}{*}{$\begin{array}{c}\text { Road Width (m) } \\
7.3\end{array}$} & \multicolumn{4}{|c|}{$\begin{array}{l}\text { Radius and Number of Curves } \\
100 \mathrm{~m} 170 \mathrm{~m} 252 \mathrm{~m} 750 \mathrm{~m}\end{array}$} \\
\hline & & & & & - & 2 & 3 & - \\
\hline Segment 2 & 4 & rural & 60 & 5.8 & 1 & 4 & - & - \\
\hline Segment 3 & 4 & urban & 40 & 7.3 & - & - & - & 5 \\
\hline Segment 4 & - & rural & 60 & 5.8 & 1 & 4 & - & - \\
\hline Segment 5 & 6 & urban & 40 & 7.3 & - & - & - & 5 \\
\hline
\end{tabular}

Roads in the rural environments were narrower than those in the urban environments, except in the first segment, which was wider than the other two rural segments (see Table 1). We did this to assess whether a decrease in road-width increased discomfort within the same road environment. Overall, rural environments were designed to have narrower roads, tighter curves, and higher speed limits (and therefore, higher resultant acceleration), along with the presence of obstacles (parked-cars and roadworks, see Figure 1). These factors were designed to increase the attentional demand of the driver at varying degrees, which could possibly induce discomfort depending on how they were negotiated by the controllers, or drivers' individual manual driving style. There were more obstacles (parked-cars, roadworks, or pedestrian refuge, see Figure 2) in the urban environments (10), when compared to the rural environments (4), to investigate whether participants' discomfort increased with the number of obstacles. 


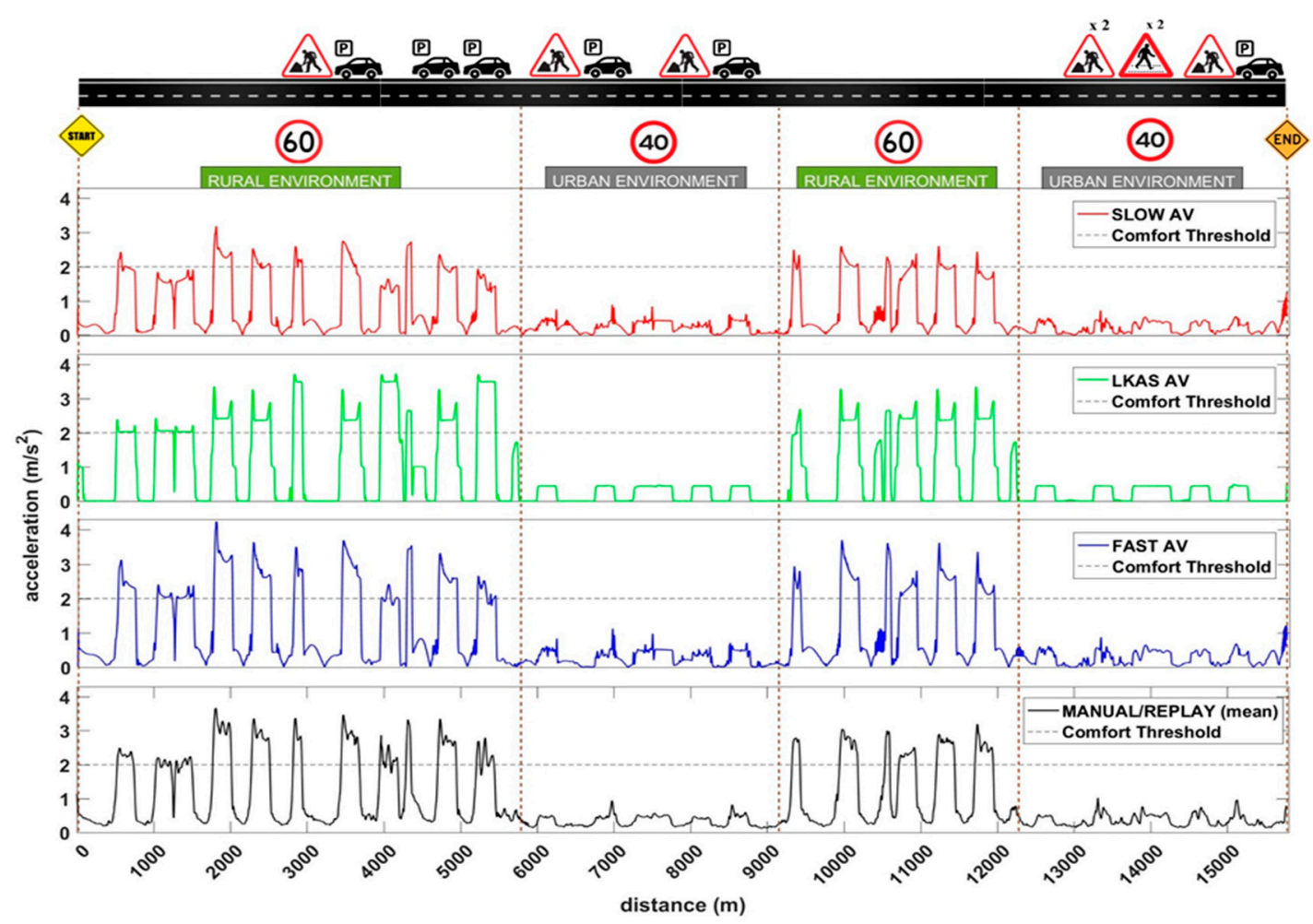

Figure 2. Resultant acceleration of the different controllers and manual driving, along with the location of obstacles across all drives, except LKAS.

\subsubsection{Experimental Design}

The five drives were counterbalanced, with the exception of the MANUAL drive, which was always the first drive for every participant, so that data could be collected for their REPLAY drive, although participants were not explicitly informed about this. As discussed in the Introduction, the SLOW and FAST controllers were modelled, based on data collected during manual driving across similar road segments in a previous HumanDrive study (see [34]). They were designed to mimic human-like driving, based on a risk model, which defined a range of acceptable vehicle states, such as velocity and lateral offset, depending on drivers' perceived risk levels in response to different road furnitures and features present in the drive, such as parked-cars or sharp curves. The FAST controller had higher velocities, compared to the SLOW controller, with a maximum difference of $4 \mathrm{~m} / \mathrm{s}$, and a minimum difference of $0.15 \mathrm{~m} / \mathrm{s}$. The driving data used to create the models (see [33]) showed that when driving at higher velocities, drivers' time to lane crossing (TLC) decreased, and, in order to maintain their preferred safety boundary, they moved further away from the road edge. Taking this knowledge into account, we increased the lateral offset of the FAST controller from the left edge of the road, at a rate of $5 \mathrm{~cm}$ for every $1 \mathrm{~m} / \mathrm{s}$ increase in relative speed, compared to the SLOW controller. The LKAS controller was a simple lane-keeping assist controller, which had a constant velocity for most parts of the drive (at the speed limit for that section), except for when the vehicle had to negotiate a curve, or when it moved from an urban to rural environment (or vice-versa). The LKAS controller mostly kept to the lane centre (even when on curves). The objective of the design of the different drives with these controllers was to understand how discomfort was affected by factors such as manual and automated driving, the behaviour of the human-like AV controllers, a conventional lane-keeping controller and the controller based on one's own driving style. The different drives and their properties are shown in Figure 2, Tables 2 and 3, which show that the LKAS controller had the highest resultant acceleration (combined lateral and longitudinal accelerations) in rural environments, whereas the SLOW controller had the lowest resultant acceleration in rural environments. The 95th percentile of resultant acceleration and lateral jerk values across all the drives in rural environments was higher 
than the suggested comfort threshold value for acceleration and jerk $\left(2 \mathrm{~m} / \mathrm{s}^{2}\right.$ and $0.9 \mathrm{~m} / \mathrm{s}^{3}$, respectively, according to [20]), whereas it was well below this threshold across all drives in the urban environments. The resultant acceleration values were mainly governed by the lateral accelerations, as the longitudinal accelerations were minimal, and within the suggested comfort threshold for longitudinal acceleration, across both environments, for all controllers.

Table 2. The 95th percentile of resultant acceleration (in $\mathrm{m} / \mathrm{s}^{2}$ ) for different drives across different road environments.

\begin{tabular}{lccccc}
\hline & MANUAL & SLOW & LKAS & FAST & REPLAY \\
\hline Rural & 3.42 & 2.34 & 3.48 & 3.20 & 3.42 \\
Urban & 0.74 & 0.47 & 0.45 & 0.57 & 0.74 \\
\hline
\end{tabular}

Table 3. The 95th percentile of absolute values of lateral jerk $\left(\mathrm{m} / \mathrm{s}^{3}\right)$ for different drives across different road environments.

\begin{tabular}{cccccc}
\hline & MANUAL & SLOW & LKAS & FAST & REPLAY \\
\hline Rural & 2.27 & 1.38 & 1.71 & 2.13 & 2.27 \\
Urban & 0.66 & 0.83 & 0.19 & 0.83 & 0.66 \\
\hline
\end{tabular}

\subsection{Subjective Discomfort Rating (Button Presses)}

For each of the automated drives, the participants heard 41 auditory beep triggers. These beeps were played immediately after the participants were exposed to any obstacles, changes in road furniture, changes in road curvature or changes in road environment. In response to these triggers, they were required to press one of two buttons on an Xbox handset, to state: "Yes, I found the behaviour to be safe/natural/comfortable" (right button) or "No, I did not find the behaviour to be safe/natural/comfortable" (left button). This response explicitly pertained to the behaviour of the car within a couple of seconds around the moment of the beep's occurrence. Additionally, participants were encouraged to give this binary input whenever they felt necessary, across each drive.

\subsection{Procedure}

Upon arrival, the participants were briefed with the description of the study, after which they were invited to sign a consent form, with an opportunity to ask questions. Three ECG electrodes were then attached to the participant's chest, and 2 EDA electrode bands were attached on the index and middle finger of their non-dominant hand. They then performed a manual familiarisation drive, where they could become accustomed to the simulator environment and vehicle controls. Participants were instructed to adhere to the posted speed limit and to obey the normal rules of the road. After each drive, the participants were given a 10-min break, during which they were asked to complete a set of subjective questionnaires relating to that drive and the controllers. The results of the subjective questionnaires are not within the scope of this paper and will not be reported here.

\subsection{Data Analysis Tools}

The ECG data was processed on Kubios HRV premium software [37]. EDA signals were pre-processed, and artefacts were removed using custom algorithms based on recommendations in [30] and [38], on MATLAB R2016a. The data were analysed using Ledalab v3.9 [39], a MATLAB-based software package.

\subsection{Statistical Analysis}

Statistical analysis was conducted on IBM SPSS Statistics 26. Shapiro Wilk's test, which showed that not all estimates across the independent variables were normally distributed, but, in general, the majority of the estimates $(>75 \%)$ were normally distributed for each of the dependent variables used. 
We judged the repeated measures ANOVA to be sufficiently robust to these issues, with only a small effect on Type I error rate [40]. For statistical significance, an $\alpha$-value of 0.05 was used, and partial eta-squared was computed as an effect size statistic. Degrees of freedom were Greenhouse-Geisser corrected when Mauchly's test showed a violation of sphericity. Pair-wise comparisons with Bonferroni corrections were used to determine the differences in different drives and road segments. Pearson's correlation coefficient was used for any correlation analyses. Data from participants 24 and 14 were classified as outliers, and the data recorded from participants 10 and 15 were of poor quality, and, hence, these were discarded for RMSSD and HR analysis. Participant 12 did not respond to the instructions given for button presses, and participant 13 had an abnormally high rate of button presses. Therefore, these participants were not considered in the subjective button press analysis.

\section{Results}

Initially, the data were analysed for five separate segments (three in rural and two in urban environments) for each of the five drives, but results for physiological metrics, and the button presses, were not statistically different between the different segments, within the same environment. Therefore, the physiological and button press data across the three rural and 2 urban segments were aggregated for analysis, with the two independent variables being drive (MANUAL, SLOW, LKAS, FAST, REPLAY) and environment (rural and urban). The dependent variables were RMSSD, mean HR and nSCR/min.

\subsection{Physiological Metrics}

To understand how the behaviour of the AV controllers and manual driving affected drivers' physiological response, and discomfort, across the different road environments, we conducted a 5 (Drive: SLOW, LKAS, FAST, MANUAL and REPLAY) $\times 2$ (Environment: rural, urban) repeated-measures ANOVA on all three physiological metrics (RMSSD, mean HR, nSCR/min). As discussed in the Introduction, previous research has shown that RMSSD values tend to decrease with an increase in discomfort, whereas mean HR and nSCR/min values tend to increase with an increase in discomfort [7,32].

There was a main effect of drive on RMSSD values, $F(2.4,45.2)=5.27, p=0.006, \eta_{p}{ }^{2}=0.22$, (Figure 3), with post-hoc tests showing significantly lower RMSSD values in the MANUAL drive, compared to the LKAS ( $p=0.007)$ and FAST $(p=0.008)$ drives. No other significant differences were found between the drives. There was no effect of environment on RMSSD, or any interactions between drive and environment.

There was a main effect of drive on drivers' mean $\operatorname{HR}, F(4,76)=6.81, p<0.001, \eta_{p}{ }^{2}=0.23$, (Figure 3), with post-hoc tests showing that drivers had significantly higher mean HR values in the MANUAL drive, compared with the FAST drive $(p=0.001)$. There were no significant differences between the other drives. There was no main effect of environment and no interactions between drive and environment. 


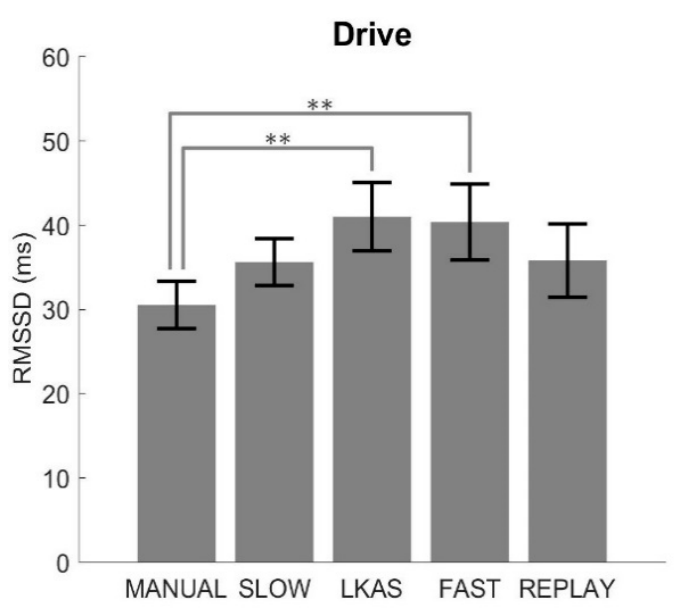

(a)

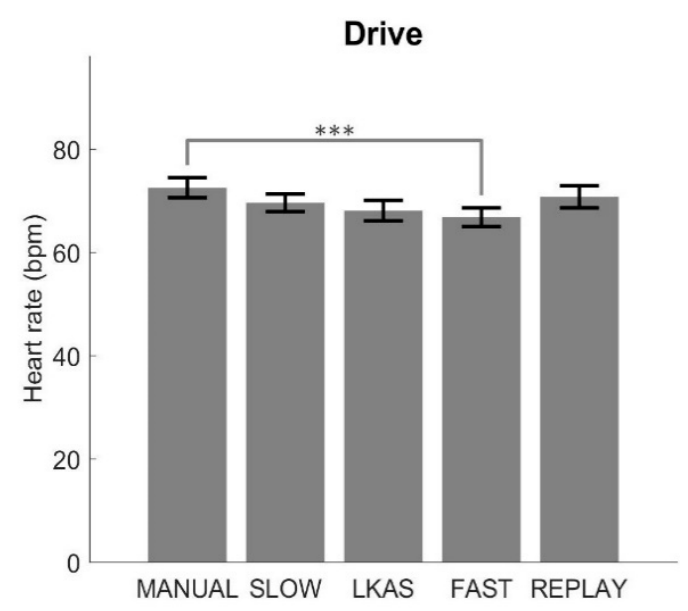

(b)

Figure 3. (a) Root mean square of successive differences (RMSSD) and (b) heart rate (HR) plots for drive. ${ }^{* *} p \leq 0.01,{ }^{* * *} p \leq 0.001$. Error bars denote s.e.

There was a main effect of drive on $\mathrm{nSCR} / \mathrm{min}, F(4,92)=4.70, p=0.002, \eta_{p}{ }^{2}=0.17$, (Figure 4a), with post-hoc tests showing that there were significantly higher nSCRs/min in the MANUAL drive, compared to the SLOW $(p=0.006)$ and REPLAY drives $(p=0.005)$. There were no other significant differences. There was also a main effect of environment on drivers' $\mathrm{nSCR} / \mathrm{min}, F(1,23)=40.54$, $p<0.001, \eta_{p}{ }^{2}=0.64$, (Figure $4 b$ ), with higher values seen in the rural environments, than the urban environments $(p<0.001)$. An interaction between drive and environment, $F(4,92)=3.37, p=0.013$, $\eta_{p}^{2}=0.13$, (Figure $\left.4 c\right)$ was also observed. Pairwise comparisons with Bonferroni corrections $(\alpha=0.002)$ revealed that, in the MANUAL drive, drivers had a significantly higher $\mathrm{nSCR} / \mathrm{min}$ while driving in rural environments, compared to the urban environments $(p<0.001)$. Additionally, within the rural environments, drivers showed significantly higher $\mathrm{nSCR} / \mathrm{min}$ values in the MANUAL drive, when compared to the SLOW $(p<0.001)$, FAST $(p<0.001)$ and REPLAY $(p=0.001)$ drives. Amongst the AV controllers, LKAS showed the largest reduction in $\mathrm{nSCR} / \mathrm{min}$ values between rural and urban environments (20.3\% reduction in mean $\mathrm{nSCR} / \mathrm{min}$ from rural to urban). 


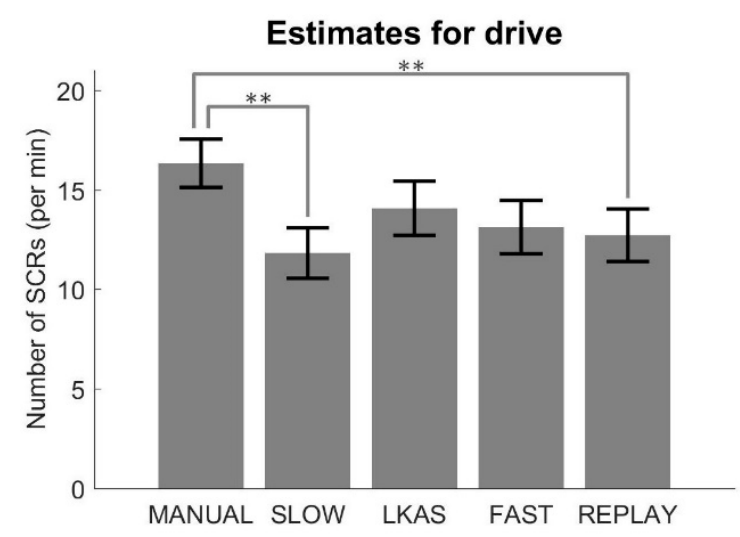

(a)

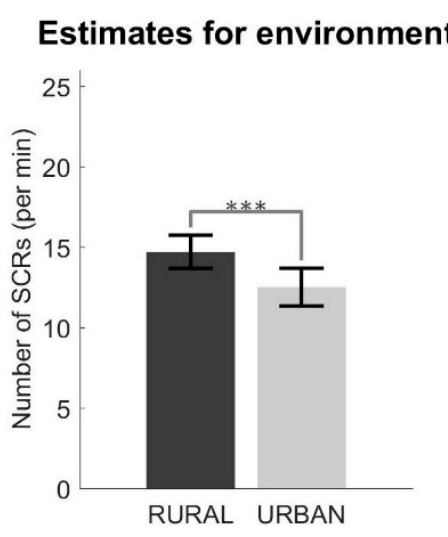

(b)

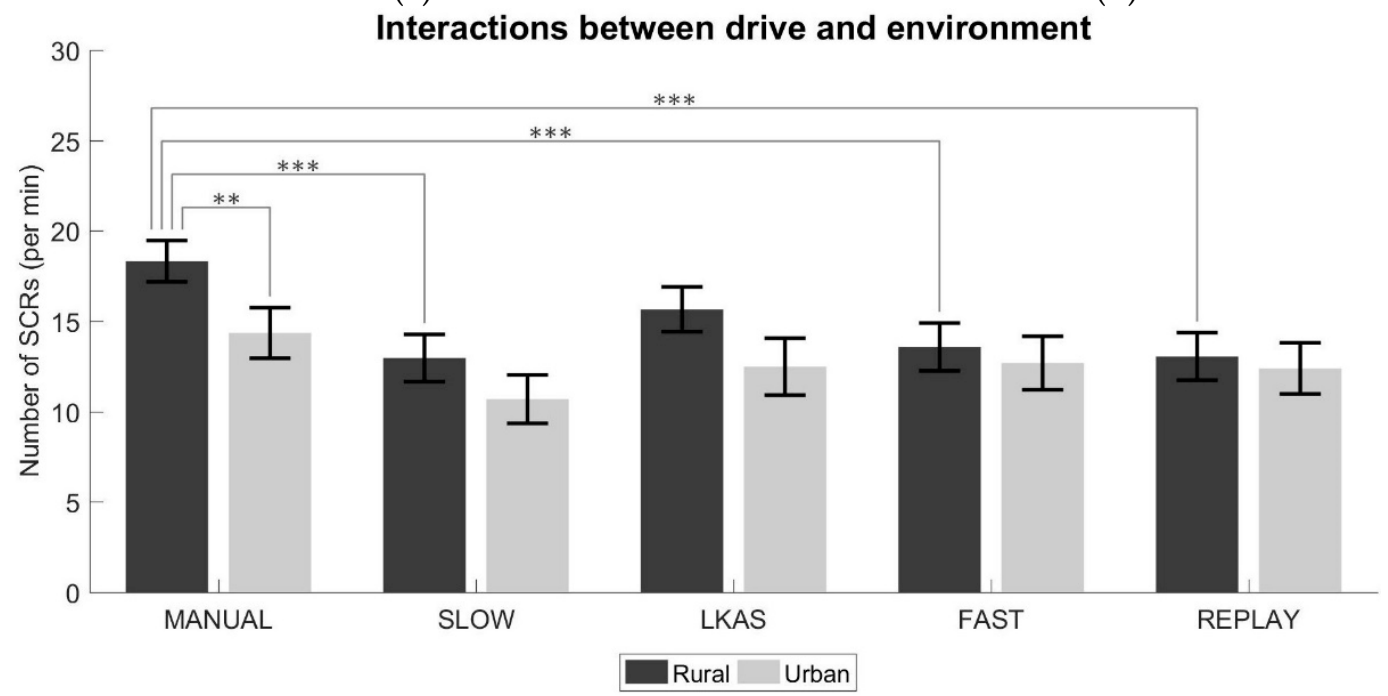

(c)

Figure 4. Number of skin conductance responses (SCRs) per minute (nSCR/min) for: (a) each drive; (b) across different environments; (c) and interaction effects. ${ }^{* *} p \leq 0.01$, ${ }^{* * *} p \leq 0.001$. Error bars denote s.e.

\subsection{Subjective Discomfort Ratings (Button Presses)}

In the previous section, we reported a comparison of drivers' physiological state during each drive. However, physiological signals are sensitive to a wide range of stimuli, and are prone to individual differences. Therefore, care must be taken when interpreting a psychological construct, such as discomfort, using physiological measures only [7]. Hence, we used data from the button presses (see Section 2.4, in the Methods section) to establish whether the changes in physiological state correlated with the participants' overall subjective discomfort rating. Correlation analysis showed that button presses and $\mathrm{nSCR} / \mathrm{min}$ were significantly positively correlated $(r(20)=0.46, p=0.04)$.

To normalise the button press data across all participants, the percentage of NO presses was calculated in relation to the total number of presses, for each road environment, in each drive. A $4 \times 2$ repeated measures ANOVA was performed on the percentage of NO presses to assess discomfort, comparing the values across the four drives (SLOW, LKAS, FAST, and REPLAY) at two different road environments (rural and urban).

ANOVA results showed no main effect of drive on participants' button presses, but there was a main effect of environment, where drivers reported a significantly higher percentage of discomfort ratings in the rural, compared to the urban environment, $F(1,21)=9.83, p=0.005, \eta_{p}{ }^{2}=0.32$ (Figure 5a). This pattern is similar to that observed for drivers' nSCR/min values, above. 


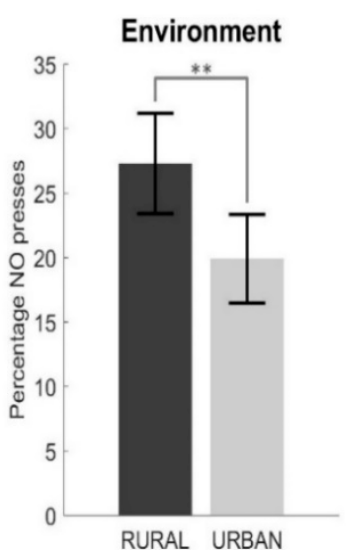

(a)

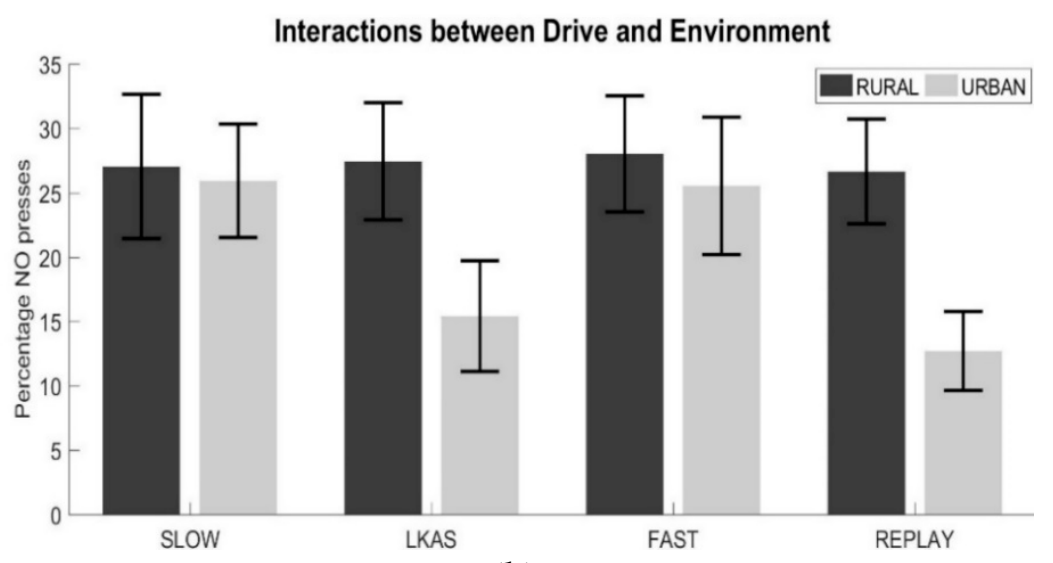

(b)

Figure 5. Percentage of NO presses: (a) across the two environments; (b) the interaction between these two factors is shown in the right graph. ${ }^{* *} p \leq 0.01$. Error bars denote s.e.

There was also an interaction effect, $F(3,63)=3.16, p=0.031, \eta_{p}^{2}=0.13$ (Figure 5b). Pair-wise comparisons with Bonferroni corrections $(\alpha=0.003125)$ did not show any significant differences between any of the drives, in each environment. Discomfort ratings were similar across all the drives in the rural environment. However, there was a $43.8 \%$ and $52.3 \%$ reduction in mean discomfort ratings for LKAS and REPLAY drives, respectively, in the urban environment, compared to their respective values in the rural environment.

\section{Discussion and Conclusions}

This study investigated driver discomfort, from a physiological perspective, and sought to establish whether drivers' physiological state changes in line with the behaviour of different automated vehicle controllers. Drivers' response in manual driving was compared to four automated drives, with each navigating through a range of road geometries and speeds, associated with urban and rural road environments.

Physiological signals can be highly subjective, and therefore individuals may respond slightly differently to a particular stimulus. Additional care must be given whilst interpreting a physiological change to a psychological construct, as a range of constructs could initiate similar psychological responses [7]. In this study, participants were pre-screened for any physiological anomalies that could occur from usage of cardiac stimulants, exercise, or any medication that they were taking. Furthermore, for EDA analysis, we used nSCR/min instead of amplitude sum of each SCRs, and the former is less susceptible to individual differences such as thickness of skin, as each event related SCR is generally initiated as a response to a particular stimuli. This, and, given the fact that our study incorporated a within-subject design, additional standardisation techniques were not applied for processing RMSSD, mean HR and nSCR/min metrics.

Results showed lower RMSSD values, and higher mean $\mathrm{HR}$ and $\mathrm{nSCR} / \mathrm{min}$ values, in the MANUAL drive, compared to at least one of the AV controllers. However, since drivers were not required to evaluate their own driving, by button presses in the MANUAL drive, it is not possible to conclude whether this difference in physiological metric between the MANUAL and automated drives reflects driver discomfort only, or rather, whether it is due to an increased physical and mental demand associated with the manual driving task, or both.

There were no significant main effects in either the physiological metrics, or button press data, between the four automated drives. This may be because overall, the drives had similar resultant acceleration profiles across the whole drive (see Figure 2.). We analysed physiological metrics and subjective button press data for each segment/environment, which were at least 2 min long. Hence, some of the instantaneous variations in controller behaviour may have produced opposing effects, 
which cancelled each other out when averaged across a larger time window. These findings are in agreement with [7], where the authors did not find any significant differences in physiological responses between their three automated drives (defensive, aggressive and replay of manual drive). Those authors attributed the lack of difference in physiological responses to high confidence interval bands in their analysis, where missing or opposite effects would have increased the confidence bands dramatically.

In contrast, there were some observable differences, both in terms of physiological metrics $\mathrm{nSCR} / \mathrm{min}$ ), and subjective button presses, for the two road environments, with the rural roads being significantly more uncomfortable than the urban environments. This increase in discomfort is likely attributed to the significantly higher resultant acceleration and jerk experienced in the rural environments, for all drives, which often crossed the $2 \mathrm{~m} / \mathrm{s}^{2}$ and $0.9 \mathrm{~m} / \mathrm{s}^{3}$ threshold for acceleration and jerk, respectively, for a comfortable driving experience, as suggested by [20]. In other words, the higher speed limits, narrower roads and tighter curves associated with the rural environments, seem to be the main cause of increased driver discomfort in this environment. Although more obstacles were present in the urban sections ( 10 vs. 4), it seems that the way these were negotiated by the vehicle in the rural sections (i.e., passed at a much higher velocity and on narrower roads), was a significant source of driver discomfort during rural environment. These findings are in line with those of [41], where the authors found higher levels of simulator sickness in high-velocity rural environments, when compared to city environments. These results also suggest that those developing automated vehicle controllers should focus on improving comfort, and thereby minimising jerk, when the vehicle is negotiating higher speed, higher acceleration, road geometries.

While the mean discomfort ratings and $\mathrm{nSCR} / \mathrm{min}$ seemed to be quite similar across all $\mathrm{AV}$ controllers in the rural environments, these were particularly low for the urban section of the LKAS (as seen in both discomfort ratings and $\mathrm{nSCR} / \mathrm{min}$ ) and REPLAY (as seen in the discomfort ratings) drives. This is likely due to the absence of any obstacles in the LKAS drive, resulting in very little variations in velocity and lateral offset (and thus, resultant acceleration). With respect to the REPLAY drive, it is likely that participants visibly recognised their own driving style and preferred this familiar behaviour during the lower speed urban environment, where their comfort threshold for acceleration forces was not breached. This was also reflected in their subjective ratings. This recognition was indeed noted by some participants, after their REPLAY drive, although not formally recorded. There seems to be incongruence in participants' physiological indicator of discomfort and perceived level of discomfort during the REPLAY drive in urban environments, indicating a bias in rating one's own driving behaviour. These findings suggest that when the resultant acceleration and jerk experienced by the driver remains well below the comfort threshold, other factors that affect discomfort, such as familiarity of the drive or presence of obstacles, become more prominent and noticeable. In contrast, when the resultant acceleration and jerk values moves above the comfort threshold, it seemingly overshadows other determinants of driver discomfort. This warrants further research into understanding drivers' comfort threshold in terms of jerk and acceleration forces, and its impact on other factors that induce discomfort to the driver.

This study was conducted on a dynamic driving simulator (see Section 2.2 for more details), and the acceleration and jerk forces experienced by the participants would be similar to that in a real-world scenario. Since acceleration and jerk were two main factors affecting discomfort, we believe a drivers' feeling of discomfort due to these forces is quite similar in a simulator and real-world environment. Johnson et al. [42] conducted a study on effect of physiological responses in fixed-based simulator vs. real-world driving and concluded that while level of immersion is at an acceptable level to elicit presence and the trends observed in physiological data during simulated driving relative to real-world driving were quite similar, the absolute physiological responses for virtual and real-world environments were significantly different. There is also the possibility of different behavioural responses by drivers in simulator, when compared to a real-world driving situation [43]. This study incorporated conventional techniques and sensors to measure drivers' physiological data, which were intrusive 
in nature. However, recent technological advancements have led to non-intrusive $[7,25]$ and even non-contact physiological sensor technologies [26], which need to be validated with on-road studies.

To conclude, there is a need to measure discomfort objectively, and in real-time, so that future AVs can adapt their driving behaviour and provide a more comfortable and pleasant driving experience for human occupants. The novelty of this study is in understanding and measuring the long-term effects of discomfort, across various road environments and a range of AV controllers, using physiological measures. This study suggests that, compared to HR variability measures, EDA-based SCR values are more sensitive to continuous changes in discomfort inducing stimuli, such as those experienced when a vehicle navigates through different geometric and speed-based scenarios. We observed a moderately positive correlation between participants' $\mathrm{nSCR} / \mathrm{min}$ and their subjective rating of discomfort. Further research may, therefore, be warranted to investigate the value of this metric for assessing real-time driver discomfort levels, which may be useful when developing more acceptable controllers for future automated vehicles.

Author Contributions: Conceptualisation, N.M., V.R., T.L., E.R.B., R.R.; data curation, V.R.; formal analysis, V.R.; funding acquisition, N.M., R.R., E.R.B.; investigation, V.R., E.P., F.H.; methodology, V.R., N.M., R.R., E.R.B., C.W., F.H., E.P.; project administration, N.M., E.R.B., R.R.; software, V.R.; supervision, N.M., T.L., M.G.L.; validation, V.R., N.M., T.L.; visualisation, V.R.; writing—original draft preparation, V.R.; writing-review and editing, V.R., N.M., T.L., M.G.L. All authors have read and agreed to the published version of the manuscript.

Funding: The work described in this paper was undertaken as part of the HumanDrive project, which is co-funded by the Centre for Connected and Automated Vehicles (CCAV) and Innovate UK, the UK's innovation agency. The lead author's Ph.D. is funded by EPSRC CASE studentship in partnership with Seeing Machines Ltd.

Acknowledgments: This paper is published with kind permission from the HumanDrive consortium: Nissan, Hitachi, Horiba MIRA, Atkins Ltd., Aimsun Ltd., SBD Automotive, University of Leeds, Highways England, Cranfield University, and the Connected Places Catapult. The data collection for this paper was feasible due to the help and technical support provided by the University of Leeds Driving Simulator (UoLDS) team.

Conflicts of Interest: The funders had no role in the design of the study; in the collection, analyses, or interpretation of data; in the writing of the manuscript, or in the decision to publish the results.

\section{References}

1. Taxonomy and Definitions for Terms Related to Driving Automation Systems for On-Road Motor Vehicles; SAE International: Warrendale, PA, USA, 2018; p. J3016.

2. ERTRAC. Automated Driving Roadmap; European Road Transport Research Advisory Council: Brussels, Belgium, 2017.

3. Carsten, O.; Martens, M.H. How can humans understand their automated cars? HMI principles, problems and solutions. Cogn. Technol. Work 2019, 21, 3-20. [CrossRef]

4. Siebert, F.W.; Oehl, M.; Höger, R.; Pfister, H.R. Discomfort in Automated Driving-The Disco-Scale. In HCI International 2013-Posters' Extended Abstracts; Stephanidis, C., Ed.; Communications in Computer and Information Science; Springer: Berlin/Heidelberg, Germany, 2013; Volume 374, ISBN 978-3-642-39476-8.

5. Beggiato, M.; Hartwich, F.; Krems, J. Using Smartbands, Pupillometry and Body Motion to Detect Discomfort in Automated Driving. Front. Hum. Neurosci. 2018, 12, 338. [CrossRef] [PubMed]

6. Slater, K. The assessment of comfort. J. Text. Inst. 1986, 77, 157-171. [CrossRef]

7. Beggiato, M.; Hartwich, F.; Krems, J. Physiological correlates of discomfort in automated driving. Transp. Res. Part F Traffic Psychol. Behav. 2019, 66, 445-458. [CrossRef]

8. Summala, H. Modelling driver behaviour in automotive environments. In Modelling Driver Behaviour in Automotive Environments: Critical Issues in Driver Interactions with Intelligent Transport Systems; Cacciabue, P.C., Ed.; Springer: London, UK, 2007; pp. 189-207. ISBN 978-1-84628-618-6.

9. Cahour, B. Discomfort, affects and coping strategies in driving activity. In Proceedings of the ECCE 2008 (European Conference on Cognitive Ergonomics), Madeira, Portugal, 16-19 September 2008; pp. 45-53.

10. Healey, J.A.; Picard, R.W. Detecting stress during real-world driving tasks using physiological sensors. IEEE Trans. Intell. Transp. Syst. 2005, 6, 156-166. [CrossRef]

11. Wertheim, A.H.; Hogema, J.H. Thresholds, Comfort and Maximum Acceptability of Horizontal Accelerations Associated with Car Driving; TNO report TM-97-C003; TNO: Soesterberg, The Netherlands, 1997. 
12. Beard, G.F.; Griffin, M.J. Discomfort caused by low-frequency lateral oscillation, roll oscillation and roll-compensated lateral oscillation. Ergonomics 2013, 56, 103-114. [CrossRef]

13. Martin, D.; Litwhiler, D. An investigation of acceleration and jerk profiles of public transportation vehicles. In Proceedings of the ASEE Annual Conference and Exposition, Pittsburgh, PA, USA, 22 June 2008.

14. Vogel, H.; Kohlhaas, R.; von Baumgarten, R.J. Dependence of motion sickness in automobiles on the direction of linear acceleration. Eur. J. Appl. Physiol. Occup. Physiol. 1982, 48, 399-405. [CrossRef]

15. Moon, S.; Yi, K. Human driving data-based design of a vehicle adaptive cruise control algorithm. Veh. Syst. Dynam. 2008, 46, 661-690. [CrossRef]

16. Bae, I.; Moon, J.; Seo, J. Toward a comfortable driving experience for a self-driving shuttle bus. Electronics 2019, 8, 943. [CrossRef]

17. Bosetti, P.; Da Lio, M.; Saroldi, A. On the human control of vehicles: An experimental study of acceleration. Eur. Transp. Res. Rev. 2014, 6, 157-170. [CrossRef]

18. Levison, W.H.; Campbell, J.L.; Kludt, K.; Bittner, A.; Harwood, D.W.; Hutton, J.; Gilmore, D.; Howe, J.G.; Chrstos, J.P.; Allen, R.W.; et al. Development of a Driver Vehicle Module for the Interactive Highway Safety Design Model; FHWA report FHWA-HRT-08-019; Federal Highway Administration: McLean, VA, USA, 2007.

19. Powell, J.P.; Palacín, R. Passenger Stability Within Moving Railway Vehicles: Limits on Maximum Longitudinal Acceleration. Urban Rail Transit 2015, 1, 95-103. [CrossRef]

20. Eriksson, J.; Svensson, L. Tuning for Ride Quality in Autonomous Vehicle Application to Linear Quadratic Path Planning Algorithm; Uppsala University: Uppsala, Sweden, 2015.

21. Thakurta, K.; Koester, D.; Bush, N.; Bachle, S. Evaluating Short and Long Term Seating Comfort; SAE Technical Paper 950144; SAE International: Warrendale, PA, USA, 1995.

22. Myers, A.M.; Paradis, J.A.; Blanchard, R.A. Conceptualizing and Measuring Confidence in Older Drivers: Development of the Day and Night Driving Comfort Scales. Arch. Phys. Med. Rehabil. 2008, 89, 630-640. [CrossRef] [PubMed]

23. Mehler, B.; Reimer, B.; Coughlin, J.; Dusek, J. Impact of Incremental Increases in Cognitive Workload on Physiological Arousal and Performance in Young Adult Drivers. Transp. Res. Rec. J. Transp. Res. Board 2009, 2138, 6-12. [CrossRef]

24. Lal, S.K.L.; Craig, A. Driver fatigue: Electroencephalography and psychological assessment. Psychophysiology 2002, 39, 313-321. [CrossRef] [PubMed]

25. McCarthy, C.; Pradhan, N.; Redpath, C.; Adler, A. Validation of the Empatica E4 wristband. In Proceedings of the 2016 IEEE EMBS International Student Conference (ISC), Ottawa, ON, Canada, 29-31 May 2016; Institute of Electrical and Electronics Engineers Inc.: Piscataway, NJ, USA, 2016; pp. 1-4. [CrossRef]

26. Kranjec, J.; Beguš, S.; Geršak, G.; Drnovšek, J. Non-contact heart rate and heart rate variability measurements: A review. Biomed. Signal Process. Control 2014, 13, 102-112. [CrossRef]

27. Mehler, B.; Reimer, B.; Wang, Y. A comparison of heart rate and heart rate variability indices in distinguishing single-task driving and driving under secondary cognitive workload. In Proceedings of the Proceedings of the Sixth International Driving Symposium on Human Factors in Driver Assessment, Training and Vehicle Design, Olympic Valley, CA, USA, 27-30 July 2011; Public Policy Center, University of Iowa: Iowa City, IA, USA, 2011; pp. 590-597.

28. Orsila, R.; Virtanen, M.; Luukkaala, T.; Tarvainen, M.; Karjalainen, P.; Viik, J.; Savinainen, M. Perceived mental stress and reactions in heart rate variability-A pilot study among employees of an electronics company. Int. J. Occup. Saf. Ergon. 2008, 14, 275-283. [CrossRef]

29. Cinaz, B.L.; Marca, R.; Arnrich, B.; Tröster, G. Monitoring of mental workload levels during an everyday life office-work scenario. Personal and Ubiquitous Computing 2013, 17, 229-239. [CrossRef]

30. Braithwaite, J.J.; Watson, D.G.; Jones, R.; Rowe, M. A Guide for Analysing Electrodermal Activity E Skin Conductance Responses (SCRs) for Psychophysiological Experiments; Behavioural Brain Sciences Centre, University of Birmingham: Birmingham, UK, 2015.

31. Dawson, M.E.; Schell, A.M.; Filion, D.L. The Electrodermal System. In Handbook of Psychophysiology, 4th ed.; Cacioppo, J.T., Tassinary, L.G., Berntson, G.G., Eds.; Cambridge University Press: Cambridge, UK, 2016; pp. 159-181. ISBN 9781107415782.

32. Foy, H.J.; Chapman, P. Mental workload is reflected in driver behaviour, physiology, eye movements and prefrontal cortex activation. Appl. Ergon. 2018, 73, 90-99. [CrossRef] 
33. Hajiseyedjavadi, F.; Merat, N.; Romano, R.; Paschalidis, E.; Boer, E. Effect of Environmental and Individual Differences on Subjective Evaluation of Human-Like and Conventional Automated Vehicle Controllers. 2020, Unpublished work.

34. Louw, T.; Hajiseyedjavadi, F.H.; Jamson, H.; Romano, R.; Boer, E.; Merat, N. The Relationship between Sensation Seeking and Speed Choice in Road Environments with Different Levels of Risk. In Proceedings of the Tenth International Driving Symposium on Human Factors in Driver Assessment, Training and Vehicle Design, Santa Fe, NM, USA, 24-27 June 2019; pp. 29-35.

35. Boer, E.R. Satisficing Curve Negotiation: Explaining Drivers' Situated Lateral Position Variability. IFAC-PapersOnLine 2016, 49, 183-188. [CrossRef]

36. Laborde, S.; Mosley, E.; Thayer, J.F. Heart rate variability and cardiac vagal tone in psychophysiological research-Recommendations for experiment planning, data analysis, and data reporting. Front. Psychol. 2017, 8, 213. [CrossRef]

37. Tarvainen, M.P.; Niskanen, J.P.; Lipponen, J.A.; Ranta-aho, P.O.; Karjalainen, P.A. Kubios HRV-Heart rate variability analysis software. Comput. Methods Prog. Biomed. 2014, 113, 210-220. [CrossRef] [PubMed]

38. Kikhia, B.; Stavropoulos, T.G.; Andreadis, S.; Karvonen, N.; Kompatsiaris, I.; Sävenstedt, S.; Pijl, M.; Melander, C. Utilizing a wristband sensor to measure the stress level for people with dementia. Sensors 2016, 16, 1989. [CrossRef] [PubMed]

39. Benedek, M.; Kaernbach, C. A continuous measure of phasic electrodermal activity. J. Neurosci. Methods 2010, 190, 80-91. [CrossRef] [PubMed]

40. Blanca, M.J.; Alarcón, R.; Arnau, J.; Bono, R.; Bendayan, R. Datos no normales: ¿es el ANOVA una opción válida? Psicothema 2017, 29, 552-557. [CrossRef]

41. Mourant, R.R.; Thattacherry, T.R. Simulator sickness in a virtual environments driving simulator. In Proceedings of the XIVth Triennial Congress of the International Ergonomics Association and 44th Annual Meeting of the Human Factors and Ergonomics Association, Ergonomics for the New Millennium, San Diego, CA, USA, 29 July 2000; SAGE Publications: Los Angeles, CA, USA, 2000; pp. 534-537.

42. Johnson, M.J.; Chahal, T.; Stinchcombe, A.; Mullen, N.; Weaver, B.; Bédard, M. Physiological responses to simulated and on-road driving. Int. J. Psychophysiol. 2011, 81, 203-208. [CrossRef]

43. Ekanayake, H.B.; Backlund, P.; Ziemke, T.; Ramberg, R.; Hewagamage, K.P.; Lebram, M. Comparing Expert Driving Behavior in Real World and Simulator Contexts. Int. J. Comput. Games Technol. 2013, 2013. [CrossRef] 